\title{
Respiratory medium and circulatory anatomy constrain size evolution in marine macrofauna
}

\author{
Noel A. Heim (D), Saket H. Bakshi, Loc Buu, Stephanie Chen, Shannon Heh, Ashli Jain, \\ Christopher Noll, Ameya Patkar, Noah Rizk, Sriram Sundararajan, Isabella Villante, \\ Matthew L. Knope, and Jonathan L. Payne (D)
}

\begin{abstract}
The typical marine animal has increased in biovolume by more than two orders of magnitude since the beginning of the Cambrian, but the causes of this trend remain unknown. We test the hypothesis that the efficiency of intra-organism oxygen delivery is a major constraint on body-size evolution in marine animals. To test this hypothesis, we compiled a dataset comprising 13,723 marine animal genera spanning the Phanerozoic. We coded each genus according to its respiratory medium, circulatory anatomy, and feeding mode. In extant genera, we find that respiratory medium and circulatory anatomy explain more of the difference in size than feeding modes. Likewise, we find that most of the Phanerozoic increase in mean biovolume is accounted for by size increase in taxa that accomplish oxygen delivery through closed circulatory systems. During the Cambrian, water-breathing animals with closed circulatory systems were smaller, on average, than contemporaries with open circulatory systems. However, genera with closed circulatory systems superseded in size genera with open circulatory systems by the Middle Ordovician, as part of their Phanerozoic-long trend of increasing size. In a regression analysis, respiratory and circulatory anatomy explain far more size variation in the living fauna than do feeding modes, even after accounting for taxonomic affinity at the class level. These findings suggest that ecological and environmental drivers of the Phanerozoic increase in the mean size of marine animals operated within strong, anatomically determined constraints.
\end{abstract}

Noel A. Heim. * Department of Geological Sciences and Stanford Earth Young Investigators, Stanford University, Stanford, California 94305, U.S.A. *Present address: Department of Earth and Ocean Sciences, Tufts

University, Medford, Massachusetts 02155, U.S.A. E-mail: noel.heim@tufts.edu

Saket H. Bakshi, Loc Buu, Stephanie Chen, Shannon Heh, Ashli Jain, Christopher Noll, Ameya Patkar, Noah Rizk, Sriram Sundararajan, and Isabella Villante. Stanford Earth Young Investigators, Stanford University, Stanford, California94305, U.S.A. E-mails: saketbakshi16@gmail.com, locbuu408@gmail.com, stephaniechen415@gmail. com, shannonheh26@gmail.com, AshliJain.college@gmail.com,chris.p.noll@gmail.com, ameyaspatkar@gmail. com,rizk.noah@gmail.com,SSundararajan@sfhs.com, bellavillante7@gmail.com

Matthew L. Knope. Department of Biology, University of Hawai 'i at Hilo, Hilo, Hawaii 96720, U.S.A. E-mail: knope@hawaii.edu

Jonathan L. Payne. Department of Geological Sciences and Stanford Earth Young Investigators, Stanford University, Stanford, CA 94305 U.S.A. E-mail: jlpayne@stanford.edu

Accepted: 14 March 2020

Data available from the Dryad Digital Repository: https://doi.org/10.5061/dryad.pk0p2ngjk

\section{Introduction}

The biovolume of the average-sized marine animal genus has increased by more than two orders of magnitude since the beginning of Cambrian time (Fig. 1) (Heim et al. 2015). The proposed causes of this size increase include both an environment more permissive of larger size due to increasing availability of oxygen in seawater (Berner et al. 2007; Dahl et al. 2010) and ecosystems more capable of supporting large animals through increasing levels of primary productivity (Tappan and Loeblich 1973; Peters 1983; Vidal and Knoll 1983; Bambach 1993; Thiebaux and Dickie 1993; Bambach 1999; Vermeij 2011, 2016). However, not all phyla and classes experienced similar trends in size (Fig. 2). For example, chordates have become substantially larger, on average, over time, whereas mollusks have been nearly constant in mean size since the Ordovician. Differences in evolutionary trajectories of marine

(C) The Author(s), 2020. Published by Cambridge University Press on behalf of The Paleontological Society. All rights reserved. This is an Open Access article, distributed under the terms of the Creative Commons Attribution licence (http:/ / creativecommons.org/licenses/by/4.0/), which permits unrestricted re-use, distribution, and reproductir ${ }^{-}$ 


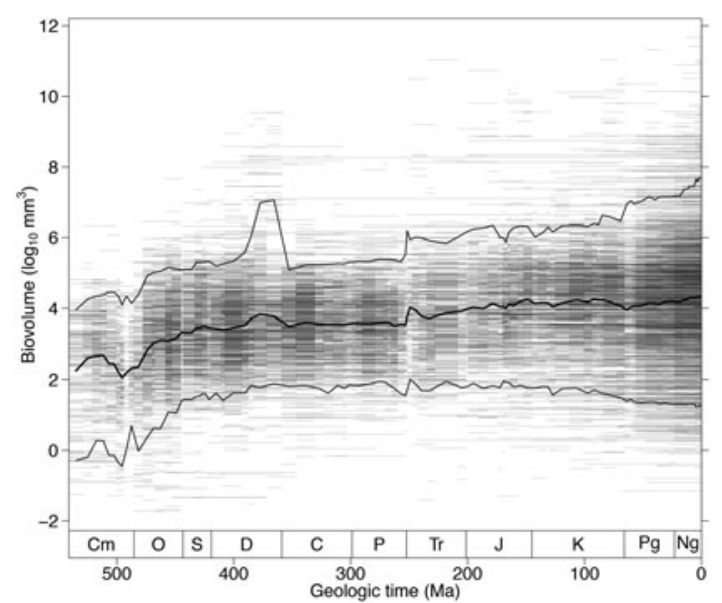

FIGURE 1. Body-size evolution across the past $541 \mathrm{Myr}$. The distribution of fossil marine animal biovolumes across the Phanerozoic is shown. The horizontal lines depict genus durations and are semitransparent so overlapping ranges appear darker. The thick black line indicates the stage-level mean body size. The thin black lines demarcate the $5^{\text {th }}$ and $95^{\text {th }}$ percentiles. $\mathrm{Cm}$, Cambrian; $\mathrm{O}$, Ordovician; $\mathrm{S}$, Silurian; D, Devonian; C, Carboniferous; P, Permian; Tr, Triassic; J, Jurassic; K, Cretaceous; Pg, Paleogene; Ng, Neogene.

animal body size across phyla suggest that size increase has been promoted, enabled, or constrained by intrinsic biological characteristics of species and does not simply represent a uniform response of the biosphere to increased availability of food or oxygen.

Physiological aspects of anatomy and ecology are the most apparent mechanisms by which phyla could differ in their ability to respond in terms of body-size evolution to changing selective pressures or environmental boundary conditions. Because trophic level is strongly associated with body size in modern marine ecosystems (Kerr and Dickie 2001; Ferrón et al. 2018), feeding ecology is one potential determinant of size evolution. Other aspects of functional ecology, such as tiering level and motility, could also have important associations with body size and differ in their distributions across phyla. Circulatory anatomy must also respond to changes in body mass, because the ratio of surface area to volume scales allometrically with body size (Schmidt-Nielsen 1984). Just as the hierarchical structuring of molecules, organelles, cells, and tissues constrains the distribution of organismal size across the full tree of life (Heim et al. 2017), differences in circulatory anatomy among phyla could play an important role in the evolution of body size in marine animals. Further, the respiratory medium animals use to acquire oxygen - whether water or air - is potentially a key physiological determinant of body size (Forster et al. 2012; Verberk and Atkinson 2013; Heim et al. 2015; Audzijonyte et al. 2019).

To assess the extent to which the differences in body size among phyla can be explained by ecological traits, circulatory anatomy, respiratory medium, or other (untested) aspects of shared evolutionary history, we compiled a comprehensive global dataset of the sizes, feeding modes, circulatory anatomies, and respiratory media of fossil and extant marine animals. We documented not only the trends in size over time as they relate to these possible predictor variables, but also the timing of size increases. Our dataset covers the major bilaterian phyla that are well represented in the fossil record and have solitary (i.e., noncolonial) adult life forms. We also consider the body-size distributions of extant meiofaunal marine animals that respire cutaneously and lack circulatory systems in relation to larger and more complex marine macrofauna.

\section{Data and Methods}

Body-Size, Anatomical, and Ecological Data

Body Size.-For the main analyses we analyzed a dataset of 13,723 fossil marine animal genera. All genera belong to the five solitary bilaterian animal phyla with diverse, wellpreserved fossil records: Arthropoda, Brachiopoda, Chordata, Echinodermata, and Mollusca. This dataset is derived and updated from the data compiled by Heim et al. (2015), and each genus has an estimate of body size. Our measure of body size is biovolume, which is estimated as the volume of an ellipsoid or cone based on the linear dimensions of the three primary body axes. Body axes were measured from published images with known scales. Each genus also has a stage-resolved stratigraphic range. Most sizes and stratigraphic ranges were taken from the Treatise on Invertebrate Paleontology, but other published sources were also used (for details, see Heim et al. 2015). 

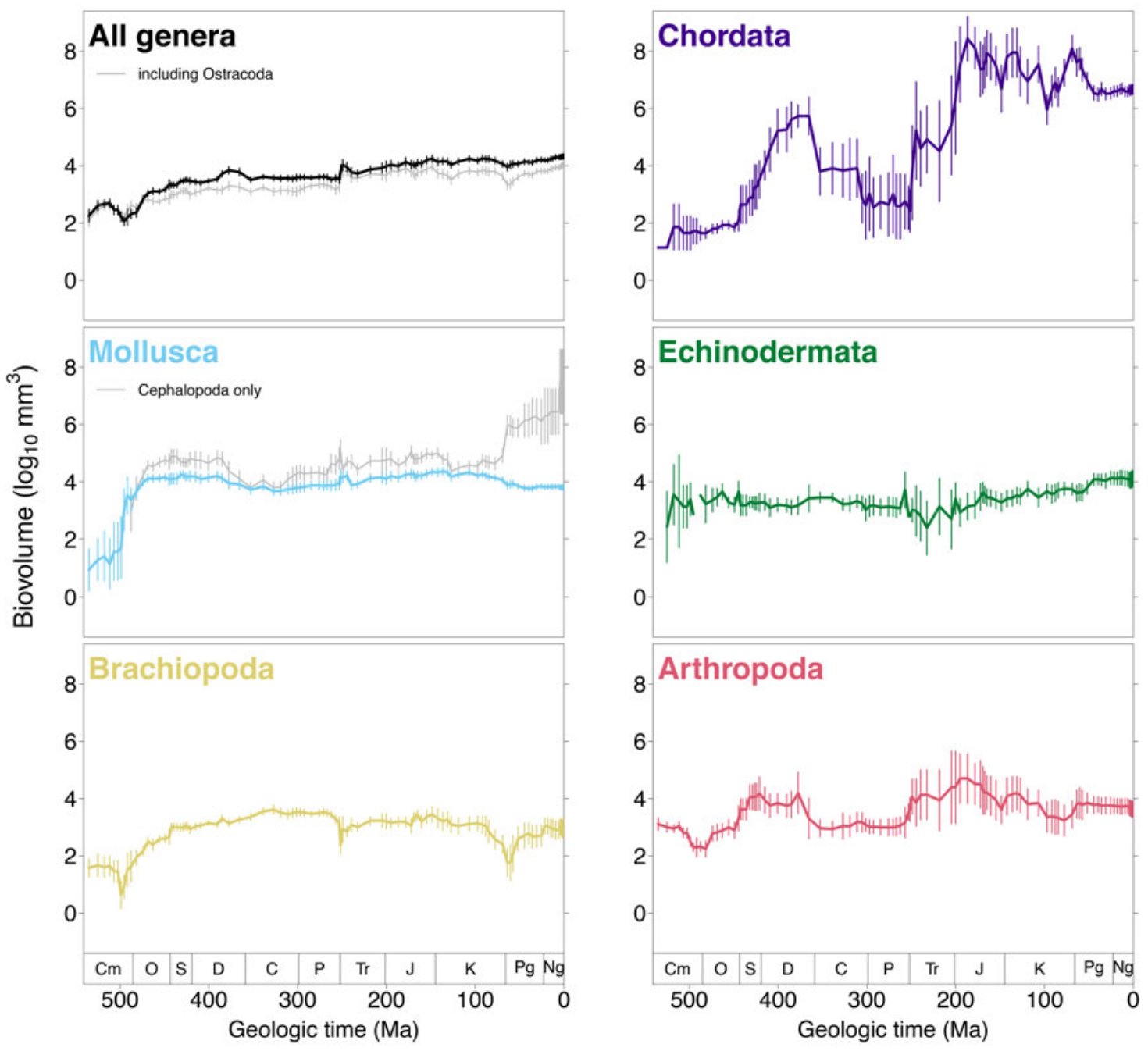

FIGURE 2. Mean size with $95 \%$ confidence intervals across the past 541 Myr within the five marine animal phyla included in this study. The mean sizes for all genera with and without the largely meiofaunal class Ostracoda are plotted in the top left panel. The mean sizes of Cephalopoda over time are plotted with the Mollusca. The timescale abbreviations are same as in Fig. 1.

Physiological Anatomy.-We considered the medium from which an animal extracts oxygen (i.e., water or air) and whether the animal has an open or closed circulatory system. In most species, the anatomical manifestation of respiratory medium is the presence of invaginated gas exchangers (i.e., internal lungs) in air breathers and evaginated gas exchangers (i.e., external gills) in water breathers. The important physiological difference between air and water breathing is that air breathers have more access to oxygen. Air holds more oxygen and is less viscous than water. Because of these properties, air breathers require much less ventilation of their respiratory organs to extract the same amount of oxygen than their water-breathing counterparts (Piiper 1982; Maina 2002). In addition, the oxygen diffusion rate from alveolar air into lung tissues is much faster than diffusion from water into gill tissues (Maina 2002, 2011; Pauly 2010). Thus, breathing air allows for much more oxygen extraction, which in turn permits larger body size by reducing the surface area constraints at larger masses (for a full explanation of the surface area to volume problem in physiology, see 
Payne et al. 2011 and references therein). The circulatory system also plays an important role in respiratory efficiency (LaBarbera and Vogel 1982) and should further constrain size increase. Open circulatory systems consist of a heart that receives oxygenated blood from gas exchangers and then pumps respiratory fluids (blood or hemolymph) into sinuses, where diffusion takes place as membranes are bathed with the respiratory fluid. While in the sinuses, the respiratory fluid delivers oxygen to cells, takes in waste products, and mixes with nonrespiratory fluids. From the sinuses, fluids are then moved back toward the gas exchangers for reoxygenation before returning to the heart. By contrast, the respiratory fluids in closed circulatory systems are fully enclosed within vessels. Oxygen-rich respiratory fluid is received in the heart from the gas exchangers, then pumped through vessels to capillaries, where diffusion of oxygen into cells and of carbon dioxide back into the fluid takes place, before the fluid is returned to the heart. Relative to open circulatory systems, closed circulatory systems move respiratory fluids with higher pressure and greater velocity (LaBarbera and Vogel 1982; Labarbera 1990; Reiber and McGaw 2009). Also, respiratory fluids within closed systems do not mix with other fluids, and oxygen-rich fluids are kept separate from oxygen-depleted carbon dioxide-rich fluids. As a result, hemolymph in animals with open circulatory systems serves an oxygen storage function as evidenced by its relatively large volume (approximately 30\% of total body water; Kanost et al. 2009). Blood within closed circulatory systems, by contrast, only constitutes approximately $5 \%$ of the animal's total mass (Lee and Blaufox 1985) and primarily serves a transport function. Because of these characteristics, animals with closed circulatory systems should have greater respiratory efficiency (Maina 2002) and, consequently, should be able to obtain larger body sizes, all else being equal.

We coded genera by their respiratory medium as being air breathers or water breathers and by their circulatory anatomy as having open or closed circulatory systems. Both of these traits were coded based on higher taxonomy, Linnaean class or order in most cases (see Supplementary Material for classification table). All genera in our dataset fell into three respiratory-circulatory modes: water breathers with open circulatory systems $(N=10,276)$, water breathers with closed circulatory systems $(N=3533)$, and air breathers with closed circulatory systems $(N=104)$. To a first approximation, there are no fully marine animals that breathe air and possess open circulatory systems. There are a few living insect species that have open circulatory systems and partially inhabit the oceans, but these taxa lack fossil records and are not considered here.

Ecology.-We applied the widely adopted ecological framework of Bush and Bambach (Bambach et al. 2007; Bush et al. 2007) to our marine animal dataset in order to code feeding mode and other potentially important ecological traits. This framework divides a theoretical ecospace along three axes, with each axis divided into six marginal states. The three axes are habitat tiering relative to the seafloor, level of motility, and mode of feeding. We used the ecological mode assignments of Knope et al. (2015) to assign modes to genera. Our primary analyses focused on feeding mode, which describes the basic feeding method, subdividing genera into six categories: (1) suspension feeders, (2) surface-deposit feeders, (3) sediment miners, (4) grazers, (5) predators, and (6) other (e.g., chemo- and photosymbiosis, parasitism). We also considered feeding mode as a binary variable of predator $(N=4778)$ versus non-predator $(N=$ 9135) to assess the impact of trophic level, given that predators are at least secondary consumers, whereas many of the non-predatory genera are predominantly suspension feeders, which are dominated by primary consumers. The full dataset is available in the online Supplementary Material.

Meiofauna.-The main focus of this study is trends in macrofauna, thus our primary analyses exclude the meiofaunal class Ostracoda. Ostracods have diverse feeding modes, and most modern species lack respiratory and circulatory systems (Vannier and Abe 1995; Bambach et al. 2007; Wilkinson et al. 2007). Unfortunately, morphological evidence of feeding mode, respiratory medium, and circulatory anatomy are rarely preserved in the fossil record of ostracods (Horne et al. 2002; Siveter 
et al. 2007, 2013). By excluding ostracods from the main analyses, we are missing the smallest animals with a robust fossil record, particularly in the post-Paleozoic, but the impact on the overall trend in mean size is minimal (Fig. 2).

Despite the absence of adequate data on fossil meiofaunal groups, the size, respiratory, and circulatory characteristics of living meiofaunal groups are instructive. We have compiled and analyzed a dataset of body sizes for 264 freeliving marine nematode genera and 138 freeliving marine podocope ostracods to complement the primary analyses. Both nematodes and ostracods respire cutaneously and lack circulatory systems (Atkinson 1980; Vannier and Abe 1995). Nematodes do not have respiratory or circulatory systems and rely on diffusion for internal oxygen delivery (Atkinson 1980). Metabolically active organisms, such as nematodes, that rely on diffusion must keep their metabolically active tissues close to oxygenated water. Generally, these organisms must be less than $1 \mathrm{~mm}$ in diameter if spherical (Krogh 1941) and have a radius less than $1 \mathrm{~mm}$ if fusiform (Atkinson 1980; Payne et al. 2011). Nematodes are able to rely on diffusion because they are roughly cylindrical with a body radius much less than $1 \mathrm{~mm}$ (Atkinson 1980). Podocopan ostracods, while slightly larger on average than nematodes, also lack respiratory and circulatory systems and conduct cutaneous respiration (Vannier and Abe 1995). Unlike nematodes, ostracods are not cylindrical, but they are able to increase their diffusion rate by ventilating sites of gas exchange by generating currents of oxygenated water using enlarged branchial plates on the cephalic maxillula (Horne 2005; Olempska et al. 2012).

\section{Statistical Methods}

To assess the associations of respiratory-circulatory mode and feeding mode on body size, we first examined size distributions of living genera. We then examined evolutionary trends in mean size within physiological and ecological groupings across the Phanerozoic to determine when and how the size differences that characterize the extant fauna developed. We have conducted analyses of extant and fossil genera separately, because combining genera that did not coexist will lead to higher genus counts for groups with high rates of taxonomic turnover. Confining comparisons of body-size distributions to standing genera at a single point in Earth history avoids this potential bias.

Modern.-For extant genera, we compared body-size distributions grouped by respiratory-circulatory mode and by feeding mode. We also compared the variation in body size within the different modes using standard deviation. To test for significant differences in mean sizes among groups, we performed an ANOVA with Linnaean class as a random effect, which allowed us to account for the effects of shared evolutionary history on body size. Linnaean class is a crude measure of phylogeny, but there is not currently a phylogeny that covers the taxonomic and temporal scope of our dataset. We applied a Tukey's honest significant differences test to determine which pairs of groups are significantly different. Finally, we calculated effect size as eta-squared $\left(\eta^{2}\right)$ using the lsr package (Navarro 2015) for R (R Core Team 2019). $\eta^{2}$ is the percent variance explained for each predictor variable in a factorial ANOVA. The sum of $\eta^{2}$ across all variables is the total $R^{2}$ (Levine and Hullett 2002; Navarro 2015).

Fossil Record.-For the extinct genera, we calculated mean size for each respiratory-circulatory mode and for each feeding mode in each of the 99 Phanerozoic stages. We also performed multiple linear regression in each stage to determine the relative strength of association of respiratory medium, circulatory system, and feeding mode with body size. Again, we included Linnaean class as a random variable to control for the possible influence of traits shared through evolutionary history without explicitly including class as a predictor variable in the analysis.

\section{Results}

Modern.-Among the three respiratory-circulatory modes for living genera, air breathers are the largest marine animals, followed by water breathers with closed circulatory systems and then water breathers with open circulatory systems (Fig. 3A, Table 1). The mean size of genera that are closed circulatory air breathers 

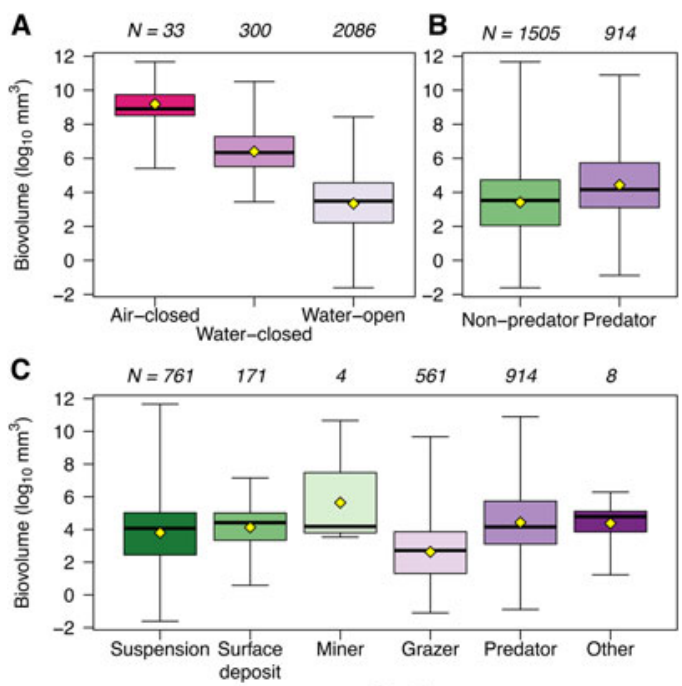

FIGURE 3. Body-size distributions of extant genera grouped by respiratory-circulatory mode and feeding mode. A, Box-and-whisker plots of $\log _{10}$ biovolume for each of the three respiratory-circulatory modes. B, Box-and-whisker plots of $\log _{10}$ biovolume for genera with predatory and non-predatory feeding modes. C, Box-and-whisker plots of $\log _{10}$ biovolume for each of the six feeding modes. Diamonds near centers of distributions are mean size. Sample sizes are given above each box plot.

is approximately three orders of magnitude larger than the mean size of genera that are closed circulatory water breathers. Further, the mean size of water-breathing genera with closed circulation is more than two orders of magnitude larger than the mean size of genera that are water breathers with open circulatory systems. This pattern of decreasing body size from air breathers with closed circulatory systems to water breathers with open circulation also holds for maximum and minimum size (Fig. 3A, Table 1). The difference in mean size between each pair of respiratory-circulatory modes is statistically significant (ANOVA: $F=$ $718, p$-value $<<0.001$ ) as are the means between all three combinations of respiratory-circulatory mode (Table 2). Thirty-seven percent of the variation in body size is explained by respiratory-circulatory mode $\left(\eta^{2}=0.37\right)$.

As body size decreases from closed circulatory air breathers to open circulatory water breathers, the variance in size increases (Table 1). Quantitative comparisons of variances using a robust Brown-Forsythe Levenetype test of equal variances show that the
TABLE 1. Summary statistics for the body sizes of extant genera grouped by respiratory-circulatory mode and feeding modes. $n$, number of genera; Min, minimum size; Max, maximum size; Mean, mean size; Median, median size; SD, standard deviation of size. Size is measured as biovolume with units of $\log _{10} \mathrm{~mm}^{3}$.

\begin{tabular}{lrrrccc}
\hline \hline Mode & $n$ & Min & Max & Mean & Median & SD \\
\hline Air-closed & 33 & 5.41 & 11.67 & 9.19 & 8.91 & 1.29 \\
Water-closed & 300 & 3.44 & 10.51 & 6.40 & 6.33 & 1.34 \\
Water-open & 2086 & -1.61 & 8.43 & 3.34 & 3.48 & 1.58 \\
Suspension & 761 & -1.61 & 11.67 & 3.80 & 4.07 & 1.80 \\
Surface deposit & 171 & 0.59 & 7.15 & 4.13 & 4.42 & 1.23 \\
Miner & 4 & 3.53 & 10.66 & 5.64 & 4.18 & 3.36 \\
Grazer & 561 & -1.10 & 9.67 & 2.63 & 2.72 & 1.69 \\
Predator & 914 & -0.89 & 10.89 & 4.43 & 4.16 & 2.00 \\
Other & 8 & 1.23 & 6.28 & 4.38 & 4.79 & 1.51 \\
\hline
\end{tabular}

variances among the groups are different (Table 3). Comparisons between the individual modes show unequal variances for some pairs. There is strong support for a difference between water breathers with open versus closed circulatory systems, slightly weaker support for differences between closed circulatory air breathers and open circulatory water breathers, and no difference in variance between air breathers and water breathers with closed circulatory systems (Table 3 ).

Living genera also show a statistically significant difference in mean size between predatory and non-predatory feeding modes (Fig. 3B), but this difference is less than the differences among groupings based on respiratory-circulatory mode. Most of the difference in size among all feeding modes is due to the relatively small sizes of grazing genera, which "scrape or nibble" (Bush et al. 2007: p. 82) food off a substrate (ANOVA: $F=70.1, p$-value $<<0.001$; Fig. 3C). The only significant size differences are between grazers and all feeding modes except "other" (only eight genera have a feeding mode of "other"; seven chemosymbiotic bivalves and one parasitic decapod) as well as between predators and suspension feeders (Table 2). Feeding mode only explains $13 \%$ of the variation in body size $\left(\eta^{2}=0.13\right)$.

We also used a two-way ANOVA to simultaneously consider the effects of respiratorycirculatory and feeding modes on extant marine animal genera with Linnaean class included as a random effect to control for phylogenetic nonindependence, and the results are consistent with the ANOVAs conducted separately 
TABLE 2. Differences in mean size between all pairwise combinations of respiratory-circulatory modes and feeding modes. Confidence limits and significance were calculated with the Tukey's honest significant differences test. Only extant genera are included in the analysis.

\begin{tabular}{|c|c|c|c|c|}
\hline Comparison & Difference & Lower CL & Upper CL & Adj. $p$-value \\
\hline Water-closed vs. air-closed & -2.79 & -3.45 & -2.13 & 0 \\
\hline Water-open vs. air-closed & -5.85 & -6.49 & -5.21 & 0 \\
\hline Water-open vs. water-closed & -3.06 & -3.28 & -2.84 & 0 \\
\hline Surface deposit vs. suspension & 0.33 & -0.11 & 0.77 & 0.28 \\
\hline Miner vs. suspension & 1.84 & -0.77 & 4.44 & 0.34 \\
\hline Grazer vs. suspension & -1.17 & -1.46 & -0.88 & 0 \\
\hline Predator vs. suspension & 0.63 & 0.37 & 0.88 & 0 \\
\hline Other vs. suspension & 0.57 & -1.28 & 2.42 & 0.95 \\
\hline Miner vs. surface deposit & 1.51 & -1.12 & 4.14 & 0.58 \\
\hline Grazer vs. surface deposit & -1.5 & -1.96 & -1.05 & 0 \\
\hline Predator vs. surface deposit & 0.3 & -0.13 & 0.73 & 0.36 \\
\hline Other vs. surface deposit & 0.25 & -1.64 & 2.13 & 1 \\
\hline Grazer vs. miner & -3.01 & -5.62 & -0.4 & 0.01 \\
\hline Predator vs. miner & -1.21 & -3.82 & 1.4 & 0.77 \\
\hline Other vs. miner & -1.26 & -4.45 & 1.92 & 0.87 \\
\hline Predator vs. grazer & 1.8 & 1.52 & 2.08 & 0 \\
\hline Other vs. grazer & 1.75 & -0.11 & 3.6 & 0.08 \\
\hline Other vs. predator & -0.05 & -1.9 & 1.79 & 1 \\
\hline
\end{tabular}

on those variables. A plurality of the variance is explained by respiratory-circulatory mode (ANOVA: $F=645, p$-value $<<0.001, \eta^{2}=0.30$ ), while much less is explained by feeding mode (ANOVA: $F=49, p$-value $<<0.001, \eta^{2}=0.05$ ).

Fossil Record.-The differences in size among extant genera with different respiratory-circulatory modes raise the question: Did the evolution of closed circulatory systems permit larger body size, or did large body size create selective pressure for more efficient respiration and circulation? The air breathers show no appreciable change in their mean size from their first appearance in the late Permian through the Pleistocene. This is not the case for the water breathers. All marine animals in the Cambrian were water breathers, and among these earliest animals were those with closed and open circulatory systems. During the Cambrian, animals with closed circulatory systems were actually

TABLE 3. Comparison of variances between extant genera grouped by respiratory-circulatory mode. Statistical tests are the results from a robust Brown-Forsythe Levene-type test of equal variances.

\begin{tabular}{lrrr}
\hline \hline & & Test & \\
Comparison & Difference & stat. & $p$-value \\
\hline All groups & $\mathrm{NA}$ & 9.54 & 0 \\
Air-closed vs. water-closed & -0.12 & 0.31 & 0.58 \\
Water-closed vs. water-open & -0.7 & 16.04 & 0 \\
Air-closed vs. water-open & -0.83 & 3.55 & 0.06 \\
\hline
\end{tabular}

smaller, on average, than animals with open circulatory systems (Fig. 4A). Following the initial evolution of small-bodied animals with closed circulatory systems, a relatively steady progression toward larger body size ensued through the remainder of the Phanerozoic. Overall, the water breathers with closed circulatory systems increased in mean size by five orders of magnitude, whereas animals with open circulatory systems increased in mean size by less than two orders of magnitude.

The evolutionary history of water breathers with closed circulatory systems shows several intervals of rapid size increases and decreases. The first episode of increase occurred early in the Ordovician Period, by which point the mean size for genera with closed circulatory systems surpassed that for genera with open circulatory systems. This episode of size increase appears to be due in part to the radiation and sustained large size of nautiloid cephalopods (Fig. 2) (Novack-Gottshall 2008; Klug et al. 2015). The first major decrease in mean size took place during the Late Devonian, a $23 \mathrm{Myr}$ interval of persistent extinction of large-bodied fish species (Sallan and Galimberti 2015), and was followed by relative stasis for the remainder of the Paleozoic. The endPermian mass extinction resulted in a shortlived increase in mean body size, reflecting selective survival of large-bodied genera, 


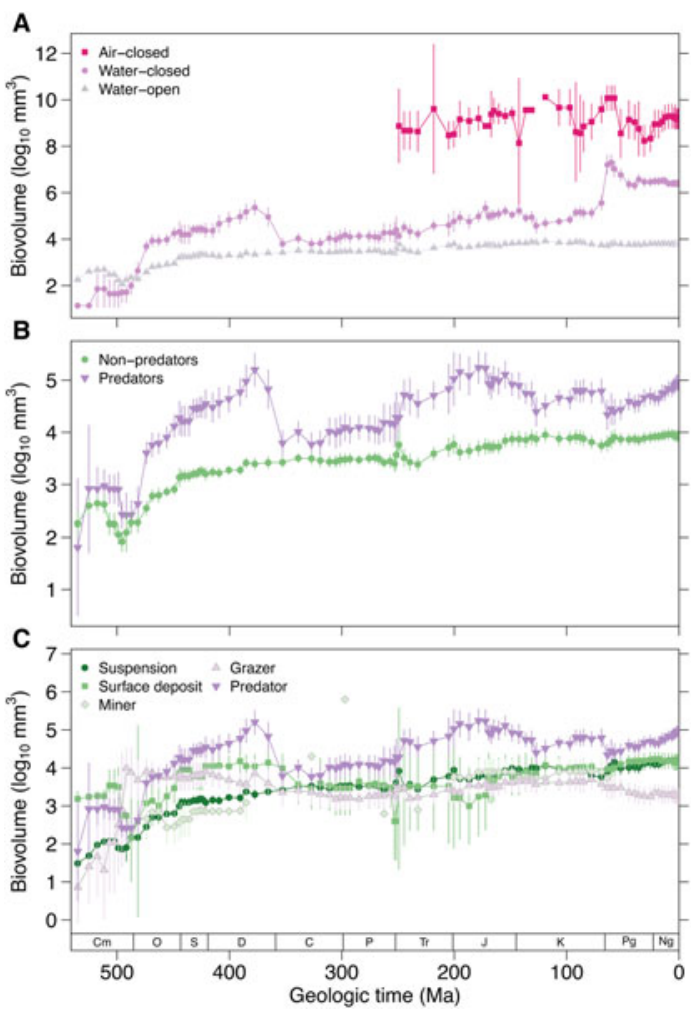

FIGURE 4. Mean trends in body size of marine animal genera across the past 541 Myr. Vertical lines are $95 \%$ confidence intervals. A, Mean size over time for each of the three respiratory-circulatory modes. B, Mean size over time for predatory and non-predatory genera. C, Mean size over time for five of the six feeding modes. The "other" feeding mode was excluded due to very small sample sizes over time.

particularly bivalves, gastropods, and cephalopods (Fig. 5A) as well as higher extinction rates in smaller-bodied classes. For example, the brachiopod classes Rhychonellata and Strophomenata are, on average, smaller than Bivalvia (Fig. 5A), but experienced much greater extinction losses (Knoll et al. 2007). Following the endCretaceous mass extinction, there was rapid increase in mean body size that corresponds to the preferential extinction of small-bodied cephalopods (i.e., ammonites were smaller, on average, than nautiloids) and bony fishes. Comparison of the mean sizes of end-Cretaceous mass extinction victims and survivors shows that in the most diverse classes, including bony fishes, cephalopods, and bivalves, survivors were much larger than their counterparts that went extinct during the Maastrichtian (Fig. 5B).
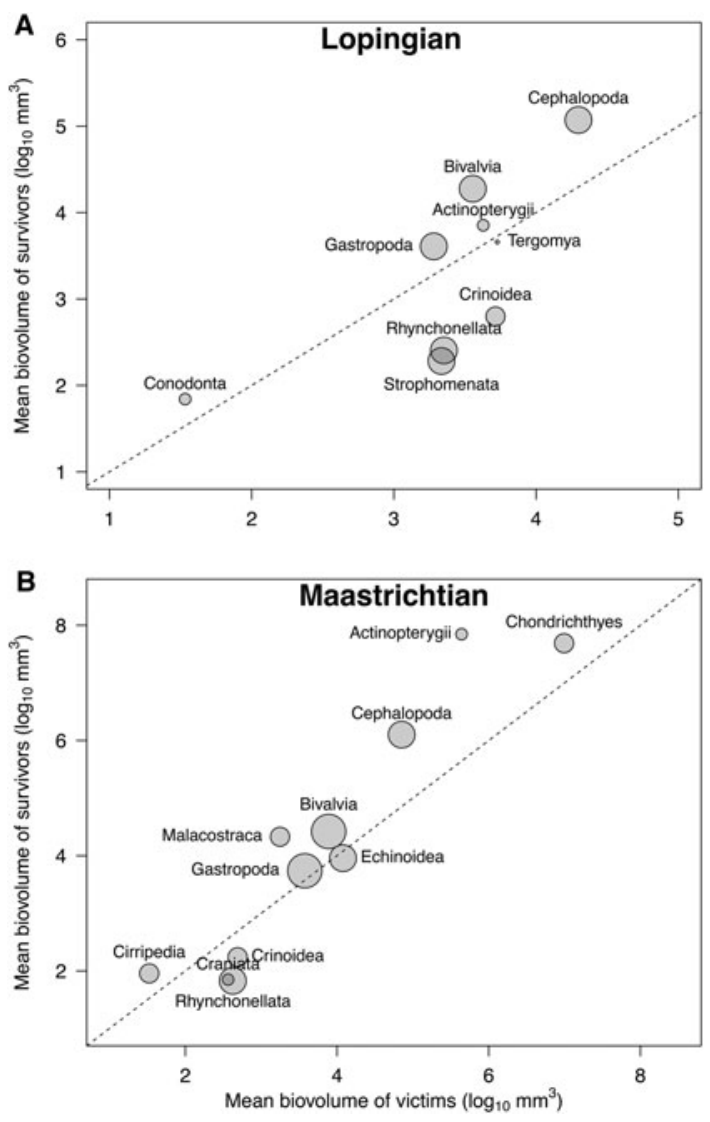

FIGURE 5. Comparisons of mean biovolumes within classes of victims and survivors of the two era-bounding mass extinctions. The sizes of the points depict relative genus richness (on $\log _{2}$ scale). The dashed lines are one-to-one lines. A, Lopingian genera classified into those that went extinct during the terminal epoch of the Permian and those that survived into the Triassic. Note that surviving brachiopods and crinoids tended to be smaller than victims of the same classes, while surviving molluscan genera tended to be larger than molluscan victims. B, Mean sizes of Maastrichtian genera, divided into those that went extinct in the terminal age of the Cretaceous and those that survived into the Danian. Note the general inverse relationship between overall body size and extinction selectivity. Victims in small-bodied classes tended to be larger than survivors, while survivors in largebodied classes tended to be larger than victims.

In contrast to the differential size trends among respiratory-circulatory modes, both predators and non-predators exhibit trends of increasing mean size across the Phanerozoic. The magnitudes of the size increases are similar in both groups, but with predators consistently exhibiting larger mean body sizes (Fig. 4B). There are no systematic trends in size histories of individual feeding modes (Fig. 4C). Mean 
sizes are somewhat volatile in the early Paleozoic, then flatten out starting in the late Paleozoic, remaining relatively constant for the remainder of the Phanerozoic. Predators and grazers separate out as the largest and smallest feeding modes, respectively, while the remaining feeding modes converge on an intermediate size.

We used a linear mixed model with Linnaean class as a random effect to predict body size as a function of respiratory medium, circulatory anatomy, and feeding mode. This model differentiates between the strength of association of those three variables with body size over time (Fig. 6). Air breathing has been associated with larger body size since the first appearance of air breathers in the marine fossil record during late Permian time. During the Cambrian, closed circulatory systems were associated with smaller body sizes. Closed circulation became associated with larger body size during the Ordovician and reached a peak in the Late Devonian. The strength of association fluctuated during the remainder of the Paleozoic and Mesozoic before increasing rapidly in the Danian and remaining quite high for the Cenozoic. In contrast, the association between feeding mode and body size has been neutral to weakly positive with predators showing a slight tendency toward larger body sizes across the Phanerozoic. The strength of the association between size and feeding mode was greater than that for respiratory-circulatory mode until the Early Devonian. Thereafter, the association between size and circulatory anatomy has been stronger, except during the Triassic (Fig. 6).

Other Aspects of Ecology.- One of the advantages of a closed circulatory system is that it enables more active lifestyles. We demonstrate this relationship by comparing the distribution of respiratory-circulatory modes within different ecological modes. We calculated the proportion of genera in each of our respiratorycirculatory modes occupying each of the 18 marginal states of the Bush et al. (2007) ecospace.

All of the air breathers are pelagic (Supplementary Fig. 1A). This result is expected, because air breathers need to access the ocean surface to breathe. However, air breathers are a relatively small portion of the overall fauna,

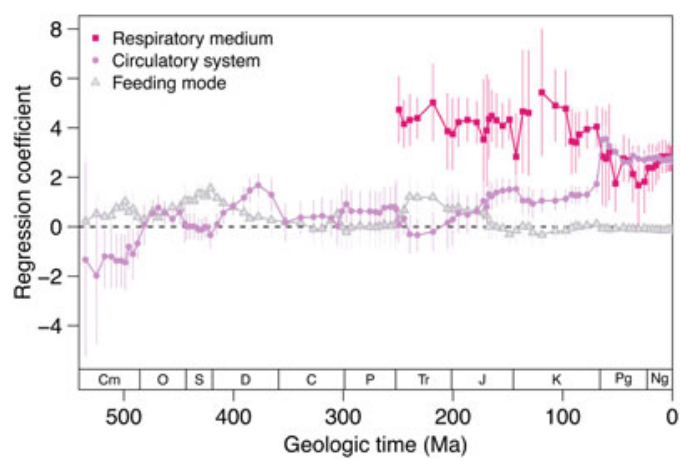

FIGURE 6. Regression coefficients for respiratory-circulatory modes and feeding mode. The multiple linear regression has the form: body size $\sim$ circulatory system + respiratory medium + feeding mode. Linnaean class is included as a random effect and all three predictor variables are binary. The feeding mode categories are non-predators (0) and predators (1). The circulatory system categories are open circulatory system (0) and closed circulatory system (1). The respiratory medium categories are water breathers (0) and air breathers (1). Positive coefficients correspond to a positive relationship between having a variable value of 1 and body size. Negative coefficients correspond to a negative relationship between having a variable value of 1 and body size. Vertical lines are 95\% confidence intervals.

and the vast majority of the pelagic animals in the dataset have closed circulatory systems. The tiering axis is partially independent of the motility axis, such that pelagic animals are entirely motile, but may be actively or passively so. Nevertheless, all of the pelagic genera with closed circulatory systems are fully motile, fast. About $20 \%$ of the semi-infaunal genera have closed circulatory systems, and a very small percentage of surficial genera have closed circulation. All of the fully infaunal and erect genera have open circulatory systems (Supplementary Fig. 1A).

The motility axis is more directly related to activity levels. Here we see a clear relationship between motility levels and the proportion of genera with closed circulatory systems (Supplementary Fig. 1B). All air-breathing genera are fully motile, fast; and all closed-circulation water breathers are motile and unattached. Of the water-breathing genera with closed circulatory systems, $80 \%$ are fully motile, fast; and nearly $20 \%$ are fully motile, slow.

Most of the air breathers are predators (Supplementary Fig. 1C), but there are a few genera distributed among the other feeding modes. 
Like the air breathers, most of the waterbreathing genera with closed circulatory systems are predators, but a few are distributed across other modes. In this ecospace framework, predators are those animals that capture prey capable of resistance (Bush et al. 2007), which implies a high degree of activity, at least for short intervals of time.

To assess the relationship between respiratory-circulatory mode and the full suite of ecological modes, we compared the body-size distributions of the three respiratory-circulatory modes within each of the three ecological axes as well as within the eight ecological modes of life with genera occupying at least two respiratorycirculatory modes. The size distributions for respiratory-circulatory modes show remarkable consistency across marginal ecological states. Along the feeding axis (Supplementary Fig. 2), air breathers are larger than water breathers with closed circulation, which are in turn larger than water breathers with open circulation. Moreover, the median size of each respiratorycirculatory mode is similar across all feeding modes. The same pattern exists for tiering level, motility level, and the ecological modes (Supplementary Fig. 2).

Higher Taxonomy.-Body size is a heritable trait (e.g., Rutledge et al. 1973; Leamy 1988; Smith et al. 1995) so systematically larger or smaller sizes in ecological or anatomical groupings that are strongly conserved across phylogeny could result from factors other than those being examined. It is therefore noteworthy that the systematic differences in size between anatomical groupings hold when the dataset is separated into different higher taxonomic groups. The Mollusca and Chordata are the two phyla with multiple respiratory-circulatory modes, and both phyla show consistent sizes across ecological modes within respiratory-circulatory modes (Supplementary Fig. 3). The rank order of sizes is consistent across all phylum-ecology combinations: the air breathers are larger than water breathers with closed circulatory systems, which are in turn larger than water breathers with open circulatory systems. However, not only is the rank order consistent, the overall sizes are approximately equal. The size distributions for air breathers grouped by marginal ecological

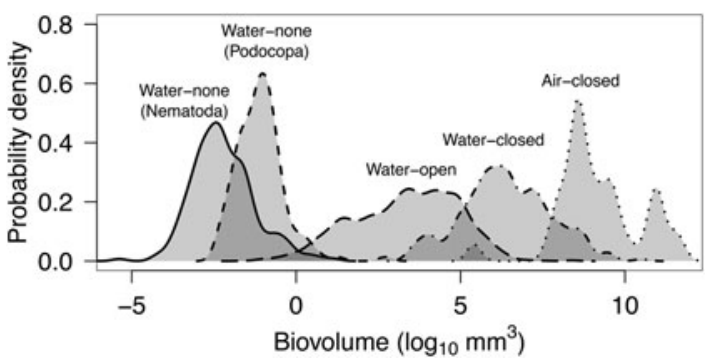

FIGURE 7. Body-size distributions of extant macrofaunal and meiofaunal genera based on respiratory-circulatory mode. Probability density distributions are plotted and were estimated by Gaussian kernel density estimation with the same bandwidth (0.214). The data used to calculate distributions for the Air-closed, Water-closed, and Water-open groups are the same as those used to generate Fig. 3A. The Water-none distributions were calculated for free-living, marine Podocopa (Class Ostracoda) and Nematoda. Podocopa and Nematoda are meiofauna that lack respiratory and circulatory systems.

state are scattered around a body size of approximately $9 \log _{10}$ biovolume units. The body-size distributions for all marginal ecological states of water breathers with closed circulatory systems, in both phyla, are scattered around a body size of approximately $6 \log _{10}$ biovolume units. Finally, the size distributions for water breathers with open circulatory systems grouped by marginal ecological state are scattered around a body size of about $4 \log _{10}$ biovolume units.

Meiofauna.-Free-living marine nematodes are smaller and have a larger variance than podocope ostracods. The right tails of the nematode and ostracod body-size distributions overlap with the left tail of the size distribution for water breathers with open circulatory systems (Fig. 7). Overall, animals that lack respiratory and circulatory systems are much smaller than animals that possess them.

\section{Discussion}

The differences in size among modern and fossil animal groups with differing respiratory-circulatory modes suggest that the efficiency of oxygen delivery to tissues is an important constraint on size evolution in marine animals. Respiratory medium and circulatory anatomy, which are among the most stable characters of body plans, were generally 
set by Cambrian time (Hall 1992). As a result, the capacity, or lack thereof, to reach very large body size was established early in the evolutionary histories of animal phyla and classes. The influence of anatomical constraint on subsequent evolutionary trends is evident in the large and progressive increase in the mean and maximum sizes of closedcirculatory-system animals relative to those with open circulatory systems.

Feeding mode has also had an important influence on body size, but its influence has operated within the constraints imposed by respiratory medium and circulatory anatomy. Over the course of the Phanerozoic, the mean sizes of predators and non-predators increased roughly in parallel, reflecting the fact that both predators and non-predators exist in all three anatomical groupings. The offset in mean size between these groups has been relatively constant across time despite the increasing mean size of each group. This pattern is congruent with both theory (Woodward et al. 2005) and observation (Cohen et al. 2003) that body size generally increases with trophic level. The differences between predators and non-predators indicate that feeding mode is important in determining body size, but the stable differences within the long-term trend in each indicate that there are additional factors that determine size evolution over time.

It is important to distinguish between extrinsic drivers of body-size change and intrinsic factors that both permit and constrain change. Increasing availability of oxygen in the environment (e.g., Nursall 1959; Rhoads and Morse 1971; Knoll and Carroll 1999; Dahl and Hammarlund 2011; Sperling et al. 2013; Boag et al. 2018) and increasing primary productivity (e.g., Bambach 1993, 1999; Vermeij 2011, 2016) are extrinsic factors that can drive bodysize evolution. None of the body-size data presented here follow the Phanerozoic trend of atmospheric oxygen concentration, which fluctuates around $20 \%$ and had maxima in the Silurian and Mississippian and minima in the Ordovician and Jurassic (Schachat et al. 2018). Primary productivity has been suggested to have increased over the Phanerozoic (Martin 1996), but this is reflected in neither growth rates (Saulsbury et al. 2019) nor ecological modes of life (McClain et al. 2018). Respiratory-circulatory modes, and internal oxygen delivery mechanisms generally, are features of body plans that determine how easy or difficult it is to achieve any given size and, effectively, limit the size extremes associated with a given body plan (e.g., Heim et al. 2017; Gearty et al. 2018). Our data show that external drivers, such as the increase in the proportion of predatory taxa, were important in body-size evolution early in the history of animals (Fig. 6), but they also demonstrate that the range of body sizes that is possible for any given clade is determined in large part by respiratory-circulatory mode and is independent of ecological mode of life.

The interpretation of respiratory-circulatory mode as a primary constraint on marine animal body size is evident by the consistency in bodysize distributions. In no categorization of genera employed here is there an observed reversal of the primary pattern: air breathers $>$ water breathers with closed circulatory systems > water breathers with open circulatory systems. Moreover, the maximum, minimum, and median sizes of genera within each respiratory-circulatory mode tend to be similar across ecological modes (Supplementary Fig. 2) and phyla (Supplementary Fig. 3).

The strong association between body size and respiratory-circulatory mode suggests constraint by hierarchical complexity of anatomy (McShea 2001; Heim et al. 2017). Considering vertical hierarchical complexity (Sterelny 1999; McShea 2001) from viruses to prokaryotes to protists to multicellular eukaryotes, "body"-size distributions of adjacent levels overlap at extreme values, but the modes are clearly distinct (Heim et al. 2017). Organisms at the extreme sizes for their level of complexity tend to have morphological or functional adaptations to cope with the challenges of extreme size. For example, $98 \%$ of the volume of Thiomargarita, the largest known prokaryote, is occupied by a vacuole, limiting the metabolically active region of the cell to a thin film where diffusion is sufficient (Schulz 2006). In this study, we restricted the main analyses to solitary bilaterian metazoans, a subset of multicellular eukaryotes. For these animals to increase in overall body size from a small 
aggregate of eukaryotic cells, they must do more than simply increase the number of somatic cells, rather they must evolve specialized cells that are organized into tissues and organs. Budd and Jensen (2000) proposed that the ancestor of animals with circulatory systems was an animal that relied on diffusion and intracellular convection to meet metabolic demands. Importantly, they argue that for this anatomical innovation to evolve, the right tail of the size distribution for diffusion-limited animals must overlap with the left tail of the size distribution of animals with circulatory systems. Our data support this model (Fig. 7), and we postulate that the most important sets of tissues and organs for increasing body size in animals are those associated with the respiratory and circulatory systems. These systems are essential for distributing oxygen from the environment to interior cells and, in a broader sense, are the systems that allow material exchange so that the entire animal can function as an integrated organism maintaining homeostasis. Our primary hypothesis is that the minimum and maximum sizes an animal can achieve are strongly constrained by physiological factors (Makarieva et al. 2005; Maurer and Marquet 2013; Smith and Lyons 2013; Ferrón et al. 2018) and ultimately related to internal oxygen transport systems.

Definitively answering the question of what sets the absolute maximum and minimum sizes of marine animals is beyond the scope of this study. However, there is anecdotal evidence that physiology and anatomical complexity exert a strong control. All else being equal, simple organ systems should be more energetically efficient to develop and maintain than complex ones. We are referring here to horizontal complexity (McShea 1996), which is the number of parts or part types (note that horizontal complexity is distinct from the vertical complexity discussed earlier). Under this framework, closed circulatory systems with veins, arteries, capillaries, multiple hearts, many-chambered hearts, and so forth are more complex than open circulatory systems. Animals with open circulatory systems are in turn more complex than animals that lack circulatory systems and rely on diffusion. If it is a general pattern that feeding efficiency increases with size (e.g., Mittelbach 1981; Ferrón et al. 2018), the expectation is that some lower size limit exists where energy expenditures exceed energy intake. In addition, the parts involved take up space in the body; thus, simple space constraints may also limit the ability of animals with complex anatomies to function at very small sizes. It must also be true that the evolution of more complex internal oxygen delivery systems is required to make large increases in size (Schmidt-Nielsen 1984; Budd and Jensen 2000).

Both nematodes and ostracods belong to the superphylum Ecdysozoa. Based on their position in the ecdysozoan phylogeny (Giribet 2016) and the limited fossil record (Poinar 2011), the ancestral nematode likely lacked circulatory and respiratory systems. The oldest unequivocal fossil nematode, Palaeonema, is Devonian in age; associated with plant fossils and "may have been a facultative plant parasite that supplemented its diet with various microorganisms" (Poinar et al. 2008: p. 12); has a maximum diameter of 23 microns; and lacks evidence of respiratory or circulatory systems (Poinar et al. 2008). The oldest known fully marine fossil nematode, Nemavermes, is Late Mississippian to Pennsylvanian in age (Schram 1979; Poinar 2011), up to $6 \mathrm{~mm}$ in diameter (Poinar 2011), and also lacks evidence of a circulatory system. (We acknowledge that freeliving marine nematodes have been reported from Upper Ordovician rocks [Knaust and Desrochers 2019], but the body fossils lack anatomical details and are diagnosed as nematodes based on overall body dimensions and associated trace fossils.) This paleontological evidence is consistent with the position of Nematoda on the ecdysozoan phylogeny within a large clade where all constituent phyla lack respiratory and circulatory systems. The putative ancestral state of nematodes is an important contrast to that of the ostracods. Unlike nematodes, ostracods are within the most derived ecdysozoan clade, Panarthropoda, in which the constituent phyla (Onychophora, Arthropoda) widely possess respiratory and circulatory systems. Evidence of respiratory and circulatory systems is common in crustaceans preserved in Cambrian Lagerstätten (Vannier et al. 1997). Of particular relevance are the 
Bradoriida, which are Cambrian stem-group crustaceans that superficially resemble ostracods (Hou et al. 1996). Bradioriids possess evidence of hemolymph sinuses on their carapace that are similar to those of Recent myodocope ostracods and malacostracans (Vannier et al. 1997). The presence of circulatory anatomy in stem-group crustaceans and in myodocope ostracods (Siveter et al. 2003, 2013) suggests the possibility that podocope ostracods, which are much smaller than other bivalved crustaceans, including myodocopes and bradoriids (Vannier and Abe 1995), have lost their circulatory systems in response to miniaturization.

The evolutionary history of animal size clearly illustrates that body-size evolution has responded to anatomical features that existed, in most cases, by Cambrian time. Early in the history of animals, feeding mode was the strongest predictor of large body size (Fig. 6), and all water-breathing animals were relatively small (Fig. 3A). This result is consistent with escalatory hypotheses for the diversification of body plans during the Cambrian explosion, in which the advent of predation led to a diversification of animal forms due to the increasing complexity of ecological interactions (Stanley 1973; Peterson et al. 2005; Marshall 2006; Butterfield 2007, 2011; Sperling et al. 2013). However, the capacity for ecological escalation to drive upward trends in the overall mean body size was quickly exhausted for animals with open circulatory systems. Animals that had closed circulatory systems, on the other hand, were able to evolve toward substantially larger body sizes, because they possessed circulatory anatomy that could distribute oxygen and other materials throughout the large bodies rapidly and efficiently (LaBarbera and Vogel 1982; Labarbera 1990; Maina 2002; Reiber and McGaw 2009). Air-breathing tetrapods further capitalized on this ability by extracting oxygen from air rather than water, where it is at higher concentration and can be taken up more rapidly and efficiently (Piiper 1982; Maina 2002; Pauly 2010).

In arguing for important constraint on size evolution from respiratory medium and circulatory anatomy, we are not arguing that animals with closed circulatory systems are better adapted to their modes of life than those with open systems, nor are we suggesting that air breathers are better adapted to life in the oceans than are water breathers. In fact, waterbreathing animals with open circulatory systems have efficient respiratory systems given their body sizes and respiratory demands (LaBarbera and Vogel 1982; McMahon and Burnett 1990; Maina 2002). Moreover, the substantial diversity of animals in each anatomical grouping across hundreds of millions of years of Earth history and across multiple higher taxa clearly indicates that each combination is highly functional within a certain range of body sizes and metabolic demands. Nor are we suggesting that respiratory-circulatory mode is the only factor that determines the body size of individual species or higher taxa. For example, closed circulatory systems may enable individuals to be more motile, and being more motile is an advantage for capturing resources, thus enabling larger maximum sizes. That said, different combinations of respiratory media and circulatory anatomies are more or less effective at different body sizes, as indicated by the relatively limited overlaps in the size distributions across anatomical groupings (Fig. 7). Because these features of body plans are highly conservative, clades are fundamentally constrained in the ranges of body sizes they can achieve based on their respiratory-circulatory mode.

In summary, the evidence that respiratory medium and circulatory anatomy are strong constraints on animal body size holds among ecological modes and among higher taxa. Feeding mode was initially more important than respiratory-circulatory anatomy in animal body size, but the importance of anatomy has increased across time, even though the basic architectural design of the respiratorycirculatory mode has not changed. This finding suggests that while there were selective pressures for size change due to ecological interactions and the physical environment may have changed in terms of favorability for body size increases (e.g., increasing oxygen), the overall capacity of phyla and classes to respond to these pressures is strongly constrained by anatomical features that were mostly established during Ediacaran or Cambrian time and have, by and large, not changed since. 


\section{Acknowledgments}

This research was supported by a CAREER grant from the U.S. National Science Foundation to J.L.P. (EAR-1151022) with additional support from the School of Earth, Energy, and Environmental Sciences at Stanford University. Supplementary figures, raw data files, and $\mathrm{R}$ code used for all analyses are permanently archived with Dryad (https://doi.org/10. 5061/dryad.pk0p2ngjk). N.A.H., M.L.K., and J.L.P designed the study; N.A.H., S.H.B., L.B., S.C., S.H., A.J., C.N., A.P., N.R., S.S., and I.V. carried out analyses. All authors contributed to writing the paper.

\section{Literature Cited}

Atkinson, H. J. 1980. Respiration in nematodes. Pp. 101-142 in B. M. Zuckerman and D. B. Dusenbery, eds. Nematodes as biological models. Academic Press, New York.

Audzijonyte, A., D. R. Barneche, A. R. Baudron, J. Belmaker, T. D. Clark, C. T. Marshall, J. R. Morrongiello, and I. van Rijn. 2019. Is oxygen limitation in warming waters a valid mechanism to explain decreased body sizes in aquatic ectotherms? Global Ecology and Biogeography 28:64-77.

Bambach, R. K. 1993. Seafood through time: changes in biomass, energetics, and productivity in the marine ecosystem. Paleobiology 19:372-397.

Bambach, R. K. 1999. Energetics in the global marine fauna: a connection between terrestrial diversification and change in the marine biosphere. Géobios 32:131-144.

Bambach, R. K., A. M. Bush, and D. H. Erwin. 2007. Autecology and the filling of ecospace: key metazoan radiations. Palaeontology 50:1-22.

Berner, R. A., J. M. VandenBrooks, and P. D. Ward. 2007. Oxygen and evolution. Science 316:557-558.

Boag, T. H., R. G. Stockey, L. E. Elder, P. M. Hull, and E. A. Sperling. 2018. Oxygen, temperature and the deep-marine stenothermal cradle of Ediacaran evolution. Proceedings of the Royal Society of London B 285:20181724.

Budd, G. E., and S. Jensen. 2000. A critical reappraisal of the fossil record of the bilaterian phyla. Biological Reviews of the Cambridge Philosophical Society 75:253-295.

Bush, A. M., R. K. Bambach, and G. M. Daley. 2007. Changes in theoretical ecospace utilization in marine fossil assemblages between the mid-Paleozoic and late Cenozoic. Paleobiology 33:76-97.

Butterfield, N. J. 2007. Macroevolution and macroecology through deep time. Palaeontology 50:41-55.

Butterfield, N. J. 2011. Animals and the invention of the Phanerozoic Earth system. Trends in Ecology and Evolution 26:81-87.

Cohen, J. E., T. Jonsson, and S. R. Carpenter. 2003. Ecological community description using the food web, species abundance, and body size. Proceedings of the National Academy of Sciences USA 100:1781-1786.

Dahl, T. W., and E. U. Hammarlund. 2011. Do large predatory fish track ocean oxygenation? Communicative and Integrative Biology 4:92-94.

Dahl, T. W., E. U. Hammarlund, A. D. Anbar, D. P. G. Bond, B. C. Gill, G. W. Gordon, A. H. Knoll, A. T. Nielsen, N. H. Schovsbo, and D. E. Canfield. 2010. Devonian rise in atmospheric oxygen correlated to the radiations of terrestrial plants and large predatory fish. Proceedings of the National Academy of Sciences USA 107:17911-17915.

Ferrón, H. G., C. Martínez-Pérez, and H. Botella. 2018. The evolution of gigantism in active marine predators. Historical Biology 30:712-716.

Forster, J., A. G. Hirst, and D. Atkinson. 2012. Warming-induced reductions in body size are greater in aquatic than terrestrial species. Proceedings of the National Academy of Sciences USA 109:19310-19314.

Gearty, W., C. R. McClain, and J. L. Payne. 2018. Energetic tradeoffs control the size distribution of aquatic mammals. Proceedings of the National Academy of Sciences USA 115:4194-4199.

Giribet, G. 2016. New animal phylogeny: future challenges for animal phylogeny in the age of phylogenomics. Organisms Diversity \& Evolution 16:419-426.

Hall, B. K. 1992. Evolutionary developmental biology. Springer, New York

Heim, N. A., M. L. Knope, E. K. Schaal, S. C. Wang, and J. L. Payne. 2015. Cope's rule in the evolution of marine animals. Science 347:867-870.

Heim, N. A., J. L. Payne, S. Finnegan, M. L. Knope, M. Kowalewski, S. K. Lyons, D. W. McShea, P. M. Novack-Gottshall, F. A. Smith, and S. C. Wang. 2017. Hierarchical complexity and the size limits of life. Proceedings of the Royal Society of London B 284:20171039.

Horne, D. J. 2005. Homology and homoeomorphy in ostracod limbs. Hydrobiologia 538:55-80.

Horne, D. J., A. Cohen, and K. Martens. 2002. Taxonomy, morphology and biology of Quaternary and living ostracoda. Pp. 5-36 in J. A. Holmes and A. R. Chivas, eds. The Ostracoda: applications in Quaternary research. American Geophysical Union, Washington, D.C.

Hou, X., D. J. Siveter, M. Williams, D. Walossek, and J. Bergström. 1996. Appendages of the arthropod Kunmingella from the early Cambrian of China: its bearing on the systematic position of the Bradoriida and the fossil record of the Ostracoda. Philosophical Transactions of the Royal Society of London B 351:1131-1145.

Kanost, M. R., V. H. Resh, and R. T. Cardé. 2009. Hemolymph. Pp. 446-449 in V. H. Resh and R. T. Cardé, eds. Encyclopedia of insects. Academic Press, Burlington, Mass.

Kerr, S. R., and L. M. Dickie. 2001. The biomass spectrum: a predator-prey theory of aquatic production. Columbia University Press, New York.

Klug, C., K. D. E. Baets, B. Kröger, M. A. Bell, D. Korn, and J. L. Payne. 2015. Normal giants? Temporal and latitudinal shifts of Palaeozoic marine invertebrate gigantism and global change. Lethaia 48:267-288.

Knaust, D., and A. Desrochers. 2019. Exceptionally preserved softbodied assemblage in Ordovician carbonates of Anticosti Island, eastern Canada. Gondwana Research 71:117-128.

Knoll, A. H., and S. B. Carroll. 1999. Early animal evolution: emerging views from comparative biology and geology. Science 284:2129-2137.

Knoll, A. H., R. K. Bambach, J. L. Payne, S. B. Pruss, and W. W. Fischer. 2007. Paleophysiology and end-Permian mass extinction. Earth and Planetary Science Letters 256:295-313.

Knope, M. L., N. A. Heim, L. O. Frishkoff, and J. L. Payne. 2015. Limited role of functional differentiation in early diversification of animals. Nature Communications 6:6455.

Krogh, A. 1941. Comparative physiology of respiratory mechanisms. University of Pennsylvania Press, Philadelphia.

LaBarbera, M. 1990. Principles of design of fluid transport systems in zoology. Science 249:992-1000.

LaBarbera, M., and S. Vogel. 1982. The design of fluid transport systems in organisms. American Scientist 70:54-60.

Leamy, L. 1988. Genetic and maternal influences on brain and body size in randombred house mice. Evolution 42:42-53. 
Lee, H. B., and M. D. Blaufox. 1985. Blood volume in the rat. Journal of Nuclear Medicine 26:72-76.

Levine, T. R., and C. R. Hullett. 2002. Eta squared, partial eta squared, and misreporting of effect size in communication research. Human Communication Research 28:612-625.

Maina, J. N. 2002. Structure, function and evolution of the gas exchangers: comparative perspectives. Journal of Anatomy 201:281-304.

Maina, J. N. 2011. Bioengineering aspects in the design of gas exchangers. Springer, New York.

Makarieva, A. M., V. G. Gorshkov, and B.-L. Li. 2005. Energetics of the smallest: do bacteria breathe at the same rate as whales? Proceedings of the Royal Society of London B 272:2219-2224.

Marshall, C. R. 2006. Explaining the Cambrian "explosion" of animals. Annual Reviews of Earth and Planetary Sciences 34:355-384.

Martin, R. E. 1996. Secular increase in nutrient levels through the Phanerozoic: implications for productivity, biomass, and diversity of the marine biosphere. Palaios 11:209-219.

Maurer, B. A., and P. A. Marquet. 2013. Processes responsible for patterns in body mass distributions. Pp. 168-186 in F. A. Smith and S. K. Lyons, eds. Animal body size: linking pattern and process across space, time, and taxonomic group. University of Chicago Press, Chicago.

McClain, C. R., N. A. Heim, M. L. Knope, and J. L. Payne. 2018. Is biodiversity energy-limited or unbounded? A test in fossil and modern bivalves. Paleobiology 44:385-401.

McMahon, B. R., and L. E. Burnett. 1990. The crustacean open circulatory system: a reexamination. Physiological Zoology 63:35-71.

McShea, D. W. 1996. Metazoan complexity and evolution: is there a trend? Evolution 50:477-492.

McShea, D. W. 2001. The hierarchical structure of organisms: a scale and documentation of a trend in the maximum. Paleobiology 27:405-423.

Mittelbach, G. G. 1981. Foraging efficiency and body size: a study of optimal diet and habitat use by bluegills. Ecology 62:1370-1386.

Navarro, D. 2015. Learning statistics with R: a tutorial for psychology students and other beginners. University of Adelaide, Australia.

Novack-Gottshall, P. M. 2008. Ecosystem-wide body-size trends in Cambrian-Devonian marine invertebrate lineages. Paleobiology 34:210-228

Nursall, J. R. 1959. Oxygen as a prerequisite to the origin of the Metazoa. Nature 183:1170-1172.

Olempska, E., D. J. Horne, and H. Szaniawski. 2012. First record of preserved soft parts in a Palaeozoic podocopid (Metacopina) ostracod, Cytherellina submagna: phylogenetic implications. Proceedings of the Royal Society of London B 279:564-570.

Pauly, D. 2010. Gasping fish and panting squids: oxygen, temperature and the growth of water-breathing animals. International Ecology Institute, Oldendorf, Germany.

Payne, J. L., C. R. McClain, A. G. Boyer, J. H. Brown, S. Finnegan, M. Kowalewski, R. A. Krause, S. K. Lyons, D. W. McShea, P. M. Novack-Gottshall, F. A. Smith, P. Spaeth, J. A. Stempien, and S. C. Wang. 2011. The evolutionary consequences of oxygenic photosynthesis: a body size perspective. Photosynthesis Research 107:37-57.

Peters, R. H. 1983. The ecological implications of body size. Cambridge University Press, Cambridge, U.K.

Peterson, K. J., M. A. McPeek, and D. A. D. Evans. 2005. Tempo and mode of early animal evolution: inferences from rocks, Hox, and molecular clocks. Paleobiology 31:36-55.

Piiper, J. 1982. Respiratory gas exchange at lungs, gills and tissues: mechanisms and adjustments. Journal of Experimental Biology 100:5-22.

Poinar, G. O. 2011. The evolutionary history of nematodes: as revealed in stone, amber and mummies. Brill, Leiden, Netherlands.
Poinar, G. O., H. Kerp, and H. Hass. 2008. Palaeonema phyticum gen. n., sp. n. (Nematoda: Palaeonematidae fam. n.), a Devonian nematode associated with early land plants. Nematology 10:9-14.

R Core Team. 2019. R: a language and environment for statistical computing, Version 3.6.0. R Foundation for Statistical Computing, Vienna, Austria. https://www.R-project.org.

Reiber, C. L., and I. J. McGaw. 2009. A review of the "open" and "closed" circulatory systems: new terminology for complex invertebrate circulatory systems in light of current findings. International Journal of Zoology 2009:301284.

Rhoads, D. C., and J. W. Morse. 1971. Evolutionary and ecologic significance of oxygen-deficient marine basins. Lethaia 4:413-428.

Rutledge, J. J., E. J. Eisen, and J. E. Legates. 1973. An experimental evaluation of genetic correlation. Genetics 75:709-726.

Sallan, L., and A. K. Galimberti. 2015. Body-size reduction in vertebrates following the end-Devonian mass extinction. Science 350:812-815.

Saulsbury, J., D. K. Moss, L. C. Ivany, M. Kowalewski, D. R. Lindberg, J. F. Gillooly, N. A. Heim, C. R. McClain, J. L. Payne, P. D. Roopnarine, B. R. Schöne, D. Goodwin, and S. Finnegan. 2019. Evaluating the influences of temperature, primary production, and evolutionary history on bivalve growth rates. Paleobiology 45:405-420.

Schachat, S. R., C. C. Labandeira, M. R. Saltzman, B. D. Cramer, J. L. Payne, and C. K. Boyce. 2018. Phanerozoic $\mathrm{pO}_{2}$ and the early evolution of terrestrial animals. Proceedings of the Royal Society of London B 285:20172631.

Schmidt-Nielsen, K. 1984. Scaling: why is animal size so important? Cambridge University Press, Cambridge.

Schram, F. R. 1979. Worms of the Mississippian Bear Gulch Limestone of central Montana, USA. Transactions of the San Diego Society of Natural History 19:107-120.

Schulz, H. N. 2006. The genus Thiomargarita. Pp. 1156-1163 in M. Dworkin, S. Falkow, E. Rosenberg, K.-H. Schleifer, and E. Stackebrandt, eds. The prokaryotes, Vol. 6. Proteobacteria: Gamma Subclass. Springer, New York.

Siveter, D. J., M. D. Sutton, D. E. G. Briggs, and D. J. Siveter. 2003. An ostracode crustacean with soft parts from the Lower Silurian. Science 302:1749-1751.

Siveter, D. J., D. J. Siveter, M. D. Sutton, and D. E. G. Briggs. 2007. Brood care in a Silurian ostracod. Proceedings of the Royal Society of London B 274:465-469.

Siveter, D. J., D. E. G. Briggs, D. J. Siveter, M. D. Sutton, and S. C. Joomun. 2013. A Silurian myodocope with preserved soft-parts: cautioning the interpretation of the shell-based ostracod record. Proceedings of the Royal Society of London B 280:20122664.

Smith, F. A., and S. K. Lyons. 2013. On being the right size. Pp. 1-10 in F. A. Smith and S. K. Lyons, eds. Animal body size: linking pattern and process across space, time, and taxonomic group. University of Chicago Press, Chicago.

Smith, F. A., J. L. Betancourt, and J. H. Brown. 1995. Evolution of body size in the woodrat over the past 25,000 years of climate change. Science 270:2012-2014.

Sperling, E. A., C. A. Frieder, A. V. Raman, P. R. Girguis, L. A. Levin, and A. H. Knoll. 2013. Oxygen, ecology, and the Cambrian radiation of animals. Proceedings of the National Academy of Sciences USA 110:13446-13451.

Stanley, S. M. 1973. An ecological theory for the sudden origin of multicellular life in the late Precambrian. Proceedings of the National Academy of Sciences USA 70:1486-1489.

Sterelny, K. 1999. Bacteria at the high table. Biology and Philosophy 14:459-470.

Tappan, H., and A. R. Loeblich. 1973. Evolution of the oceanic plankton. Earth-Science Reviews 9:207-240.

Thiebaux, M. L., and L. M. Dickie. 1993. Structure of the body-size spectrum of the biomass in aquatic ecosystems: a consequence of allometry in predator-prey interactions. Canadian Journal of Fisheries and Aquatic Sciences 50:1308-1317. 
Vannier, J., and K. Abe. 1995. Size, body plan and respiration in the Ostracoda. Palaeontology 38:843-873.

Vannier, J., M. Williams, and D. J. Siveter. 1997. The Cambrian origin of the circulatory system of crustaceans. Lethaia 30:169-184.

Verberk, W. C. E. P., and D. Atkinson. 2013. Why polar gigantism and Palaeozoic gigantism are not equivalent: effects of oxygen and temperature on the body size of ectotherms. Functional Ecology 27:1275-1285.

Vermeij, G. J. 2011. Shifting sources of productivity in the coastal marine tropics during the Cenozoic era. Proceedings of the Royal Society of London B 278:2362-2368.
Vermeij, G. J. 2016. Gigantism and its implications for the history of life. PLoS ONE 11:e0146092.

Vidal, G., and A. H. Knoll. 1983. Proterozoic plankton. Geological Society of America Memoir 161:265-278.

Wilkinson, I., P. Wilby, P. Williams, D. Siveter, and J. Vannier. 2007. Ostracod carnivory through time. Pp. 39-57 in A. M. T. Elewa, ed. Predation in organisms. Springer, New York. Woodward, G., B. Ebenman, M. Emmerson, J. M. Montoya, J. M. Olesen, A. Valido, and P. H. Warren. 2005. Body size in ecological networks. Trends in Ecology and Evolution 20:402-409. 